\title{
Personal name Index
}

Absalon, archbishop of Lund $81,83-85,87$

Adam of Bremen 46

Aegidius Albertinus 160

FInoth 77-80, 83-84, 87

Aeneas 4

Alcuin 75

Alexander, stonemason 62

Anders Sunesen, archbishop of Lund 85

Andersson, Roger 129

André Cardinal Destouches 159

Anna Margrethe Lasson 165, 166, 168

Anselm, archbishop of Canterbury 72, 78

Antoine Houdar de La Motte 159

Ari Porgilsson inn fródi 15, 175-178, 183, 187-188, 201, 206, 209-211

Árni Magnússon 95, 183, 188-189, 199-201, 203-206, 210-211

Árni vaði, archbishop 97

Asser, archbishop of Lund 78

Assmann, Jan 179

Bampi, Massimiliano 3

Bartlett, Robert 6

Bäuml, Franz H. 79

Bengt, bishop of Skara 58

Bergr Sokkason 73, 95-96

Bernardo Tasso 160

Björn, rune carver 58

Björn Jónsson á Skardsá 176

Blennow, Anna 3, 13, 54-55

Boeck, Simon Skovgaard 163

Bruun, Henry 129

Brynolf, bishop of Skara 46

Bumke, Joachim 182

Bussæus, Andreas 196, 198, 200

Buzon, Christine de 159

Canute IV, Danish king $26,75-79$

Canute Lavard, Danish prince 80

Cerquiglini, Bernard 164

Christen Worm 193-196, 198, 201

Chrétien de Troyes 106-108, 110-111

Christoph Martin Wieland 160

Clanchy, Michael 127-128

Clunies Ross, Margaret 178-179
Cooper, Helen 103

Copeland, Rita 5

Curtius, Ernst Robert 2

Dante Alighieri 120

Eisenstein, Elizabeth 74

Elin/Helena, saint 46

Ericus Schroderus 160

Even-Zohar, Itamar 8

Eyjólfur Björnsson 95

Fowden, Garth 10

Francesco Petrarca 83, 120-121

Gabriel Voigländer 166

Garci Rodríguez de Montalvo 158

Genette, Gérard 177, 179-180, 193

Geoffrey Chaucer 117, 120

Georg Friedrich Händel 159

George, saint 108, 110

Gérard of Quierzy 93-94

Giovanni Boccaccio 83, 120

Glauser, Jürg 164, 173

Green, David H. 5

Gropper, Stefanie 3

Grotowski, Piotr 108

Guðbrandur Vigfússon 97

Guðmundur Arason, Icelandic bishop

and saint 93,99

Guibert of Nogent 94-95, 98

Gyrðir Îvarsson, bishop of Skálholt 98, 99

Hagland, Jan Ragnar 129, 138

Hákon Hákonarson IV, Norwegian king 111

Halldór Hermannsson 182

Haquinus, stonemason 61-62

Harald Hecheson (or Häggesson) 136

Harald, magister, stonemason 59-60, 62, 66

Hedström, Ingela 14

Hélinand of Froidmont 95-96

Hériman of Tournai, see Hermann of Laon

Hermann of Laon 94-95

Hermann, Pernille 179

Hjalti Snær Ægisson 13 
Hødnebø, Finn 129

Hogenskild Bielke 160

Honoré d'Urfé 166

Honorius Augustodunensis 33

Horn, Anna Catharina 3

Imer, Lisbeth M. 13, 24

Irvine, Martin 5

Jacob Mörk 165

Joachim Beccau 159

Johan in Vrångfall 135

Johann Rist 166

Johannes Ewald 166

Johansson, Karl G. 3

Jón, Icelandic bishop and saint 93, 99

Jón Erlendsson 176, 180, 182-183, 187-190, 195-196, 198, 200-201, 204, 207, 209-210

Jón Halldórsson 118-122

Jón Loftsson 111

Jón Ólafsson úr Grunnavík 201, 204-205

Jón Sigurdsson 201

Jón Sigurdsson 205, 207

Jónas Kristjánsson 174

Jorge Coci 158

Jörundur, archbishop of Niðarós 96-97

Jörundur Porsteinsson, bishop of Hólar 97

Juan de la Cerda 160

Källström, Magnus 20

Keane, Webb 30

Ketill, Icelandic bishop 174

Kleivane, Elise 3

Knirk, James 20

Knudsen, Anders Leegaard 129

Knut Eriksson, king 46

Knut Magnusson, Danish king 47

Kolskeggr inn vitri 176, 193

Kujawinski, Jakub 71

Lárentíus Kálfsson 96-97

Lehtonen, Thomas M. S. 5

Leonardo Bruni 88

Liepe, Lena 110

Lindow, John 178-179

Ljung, Cecilia 49

Lucía Megías, José Manuel 158

Ludvig Holberg 161, 166
Mambrino Roseo da Fabriano 159

Marteinn H. Sigurðsson 121

Michele Tramezzino 159

Miguel Cervantes 160

Møllmann, Bernhard 201, 205

Mortensen, Lars Boje 4-5, 71, 84

Mostert, Marco 126

Müller Wille, Klaus 163

Neri, Stefano 159

Nicolas de Herberay Des Essarts 159

Niskanen, Samu 13

Oddur Pórarinsson 111

Olof Bengtsson, scribe 137

Olof Skötkonung, Swedish king 46, 59

Ormr Snorrason 113

Otto Thott 161

Palm, Rune 47

Palumbo, Alessandro 13

Parkes, Malcolm B. 138

Partonopeu de Blois 112-116, 122

Paulli, Richard 161

Pettersson, Jonatan 3

Pietro Torri 159

Platen, Magnus von 166

Præstgaard, Lise Marie 114

Ralph of Battle 82

Randalín Filippusdóttir 111

Reinhard Keiser 159

Remigius, saint 98

Richter, Anna Katharina 14

Robert of Ely $80,83-84,87$

Rohrbach, Lena 163, 175

Root, Robert K. 74

Rösli, Lukas 14

Rubenstein, Jay 94

Ruy Páez de Ribera 159

Sæmundr Sigfússon inn fródi 174

Sæmundur Jónsson 111

Saxo Grammaticus 47, 71, 74, 80-87

Sebastiano Biancardi 159

Sif Rikhardsdottir 14, 112, 167-168

Sigfrid, English bishop 46

Sigmund Feyerabend 159

Sigraiv, stonemason 62 
Snorri Sturluson 176

Sobecki, Sebastian 103

Søren Terkelsen 166

Spurkland, Terje 126

Stangerup, Hakon 161

Steenholt, Rikke Olesen 32

Stefán Karlsson 129-130, 132, 136

Steinunn Kristjánsdóttir 108, 111

Stock, Brian 5, 126

Stoklund, Marie 23

Ström, Annika 61

Sveinbjörn Rafnsson 175

Sven Aggesen 72, 80-81, 84, 87

Theodoric the Great (Dietrich von Bern) 108

Thomas de Bretagne 118

Thurgot, bishop of Skara 46

Torfi Jónsson 183

Törngren, Anders 165

Turville-Petre, Gabriel 95, 174

Unger, Carl R. 93, 95

Urban Hiärne 166
Valdemar I, Danish king 81, 84

Valdemar II, Danish king 85

Vincent of Beauvais 95

Wallace, David 5, 10, 103-104, 122

Widding, Ole 95

Wiktorsson, Per-Axel 137

Willibrord, saint 76

Zumthor, Paul 164

Pórður Porláksson, Icelandic bishop 189, 193-194, 198

Porgeir Guðmundsson 206

Porlákr, Icelandic bishop 174

Porlákur, Icelandic bishop and saint 93

Pormódur Torfason 183

Póroddr rúnameistari 175

Porsteinn Helgason 206 
